\title{
Application of Adomian Modified Decomposition Method to Free Vibration Analysis of Rotating Beams
}

\author{
Qibo Mao \\ School of Aircraft Engineering, Nanchang HangKong University, 696 South Fenghe Avenue, Nanchang 330063, China \\ Correspondence should be addressed to Qibo Mao; qibo_mao@yahoo.com
}

Received 7 January 2013; Revised 23 February 2013; Accepted 23 February 2013

Academic Editor: Safa Bozkurt Coskun

Copyright (C) 2013 Qibo Mao. This is an open access article distributed under the Creative Commons Attribution License, which permits unrestricted use, distribution, and reproduction in any medium, provided the original work is properly cited.

\begin{abstract}
The Adomian modified decomposition method (AMDM) is employed in this paper for dynamic analysis of a rotating EulerBernoulli beam under various boundary conditions. Based on AMDM, the governing differential equation for the rotating beam becomes a recursive algebraic equation. By using the boundary condition equations, the dimensionless natural frequencies and corresponding mode shapes can be easily obtained simultaneously. The computed results for different boundary conditions as well as different offset length and rotational speeds are presented. The accuracy is assured from the convergence and comparison published results. It is shown that the AMDM offers an accurate and effective method of free vibration analysis of rotating beams with arbitrary boundary conditions.
\end{abstract}

\section{Introduction}

The rotating Euler-Bernoulli beams have been the subject of numerous investigations because they are widely used in various aeronautical, robotic, and helicopter blade and wind turbine engineering fields. The free vibration analysis of rotating beams has been extensively studied by many researchers [1-10] with great success. Different numerical or analysis methods such as differential transformation method $[1,2]$, the Frobenius method [3], finite element method [4,5], and dynamic stiffness method [6] have been used in solving free vibration problems of such structures. References in $[4,5]$ give an exhaustive literature survey on the free vibration analysis of rotating beams. References in [11-13] discussed dynamic response of rotating beams with piezoceramic actuation and localized damages. No attempt will be made here to present a bibliographical account of previous work in this area. Few selective recent papers [1-10] which provide further references on the subject are quoted.

Until now, most of the vibration analysis of rotating beams has been limited to classical boundary conditions (i.e., which are either clamped, free, simply supported, or sliding). In practice, however, the characteristics of a test structure may be very well depart from these classical boundary conditions. In this paper, a relatively new computed approach called Adomian modified decomposition method (AMDM) [14-21] is used to analyze the free vibration for the rotating Euler-Bernoulli beams under various boundary conditions, rotating speeds, and offset lengths. The AMDM is a useful and powerful method for solving linear and nonlinear differential equations. The goal of the AMDM is to find the solution of linear and nonlinear, ordinary, or partial differential equation without dependence on any small parameter like perturbation method. The main advantages of AMDM are computational simplicity and do not involve any linearization, discretization, perturbation, or unjustified assumptions which may alter the physics of the problems [14]. In AMDM, the solution is considered as a sum of an infinite series and rapid convergence to an accurate solution [15]. Recently, AMDM has been applied to the problem of vibration of structural and mechanical systems, and this method has shown reliable results in providing analytical approximation that converges rapidly [16-21].

Using the AMDM, the governing differential equation for the rotating beam becomes a recursive algebraic equation [14-17]. The boundary conditions become simple algebraic frequency equations which are suitable for symbolic computation. Moreover, after some simple algebraic operations on these frequency equations, we can obtain the natural frequency and corresponding closed-form series solution of 


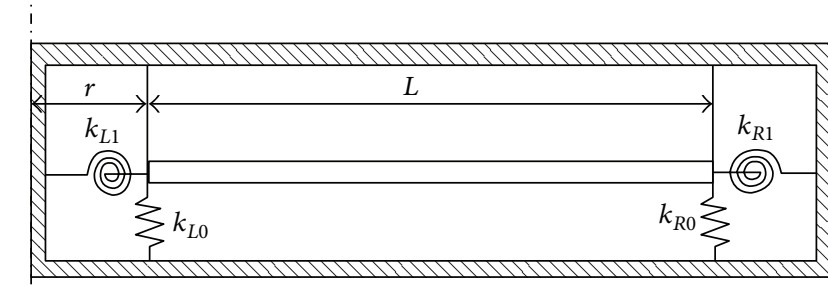

$\overbrace{\substack{i \\ i}} \Omega$

FIGURE 1: A rotating beam elastically restrained at both ends.

mode shape simultaneously. Finally, some numerical examples are studied to demonstrate the accuracy and efficiency of the proposed method.

\section{AMDM for the Rotating Beams}

Consider the free vibration of a rotating Euler-Bernoulli beam with length $L$, constant thickness $h$, and width $b$, as shown in Figure 1. The partial differential equation describing the free vibration of a rotating beam is as follows $[1,2]$ :

$$
E I \frac{d^{4} w(x, t)}{d x^{4}}+\rho A \frac{d^{2} w(x, t)}{d t^{2}}-\frac{d}{d x}\left[T(x) \frac{d w(x, t)}{d x}\right]=0,
$$

where $E$ is Young's modulus, $I(x)=b h^{3} / 12$ is the crosssectional moment of inertia of the beam, $A=b h$ is the crosssectional area, and $\rho$ is the density of the beam. $T(x)$ is the axial force due to the centrifugal stiffening and is given by the following:

$$
\begin{aligned}
T(x) & =\int_{x}^{L}\left[\rho A \Omega^{2}(r+x)\right] d x \\
& =0.5 \rho A \Omega^{2}\left(L^{2}+2 r L-2 r x-x^{2}\right),
\end{aligned}
$$

where $\Omega$ is the angular rotating speed of the beam and $r$ is offset length between beam and rotating hub.

According to modal analysis approach (for harmonic free vibration), the $w(x, t)$ can be separable in space and time:

$$
w(x, t)=\phi(x) e^{i \omega t}
$$

where $i=\sqrt{-1}, \phi(x)$ and $\omega$ are the structural mode shape and the natural frequency, respectively.

Substituting (3) into (1), then separating variable for time $t$ and space $x$, the ordinary differential equation for the rotating beam can be obtained:

$$
E I \frac{d^{4} \phi(x)}{d x^{4}}-\frac{d}{d x}\left[T(x) \frac{d \phi(x)}{d x}\right]-\rho A \omega^{2} \phi(x)=0 .
$$

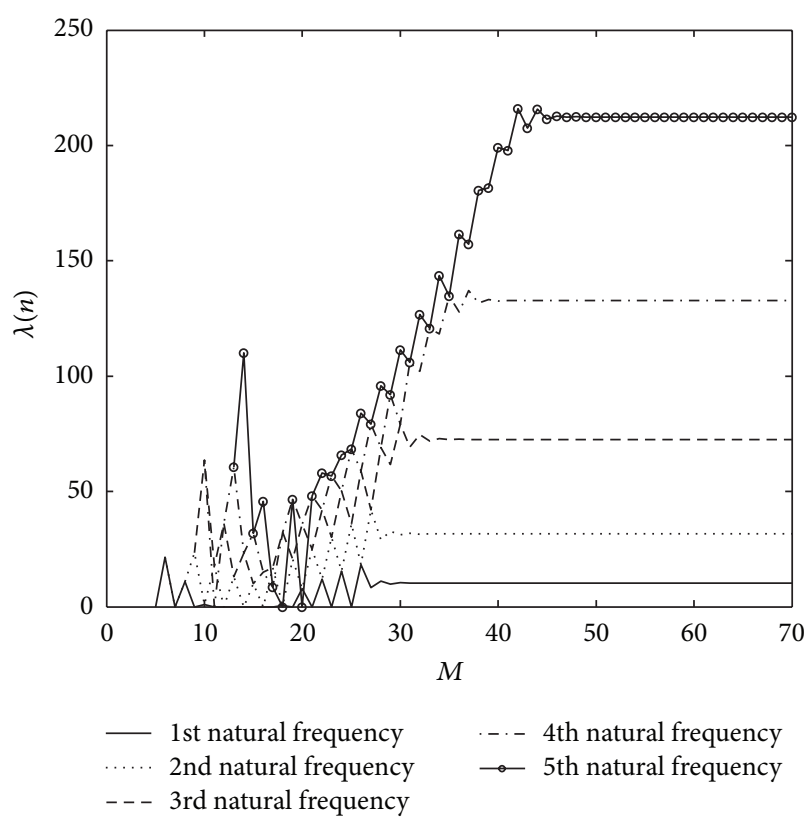

FIGURE 2: The first five dimensionless natural frequencies $\lambda(n)$ as the function of the series summation limit $M$.

Substituting (2) into (4), then rewriting (4) in dimensionless form,

$$
\begin{aligned}
& \frac{d^{4} \Phi(X)}{d X^{4}}-0.5 U^{2}(1+2 R) \frac{d^{2} \Phi(X)}{d X^{2}} \\
& \quad+U^{2} R \frac{d}{d X}\left[X \frac{d \Phi(X)}{d X}\right]+0.5 U^{2} \frac{d}{d X}\left[X^{2} \frac{d \Phi(X)}{d X}\right] \\
& \quad-\lambda^{2} \Phi(X)=0,
\end{aligned}
$$

where $X=x / L, \Phi(X)=\phi(x) / L, R=r / L, U=\sqrt{\rho A \Omega^{2} L^{4} / E I}$ is the dimensionless rotating speed and $\lambda=\sqrt{\rho A_{0} \omega^{2} L^{4} / E I}$ is the dimensionless natural frequency.

According to the AMDM [11-18], $\Phi(X)$ in (5) can be expressed as an infinite series:

$$
\Phi(X)=\sum_{m=0}^{\infty} C_{m} X^{m},
$$

where the unknown coefficients $C_{m}$ will be determined recurrently.

Impose a linear operator $G=d^{4} / d X^{4}$, then the inverse operator of $G$ is therefore a 4 -fold integral operator defined by the following:

$$
\begin{gathered}
G^{-1}=\iiint \int_{0}^{x}(\cdots) d X d X d X d X, \\
G^{-1} G[\Phi(X)]=\Phi(X)-C_{0}-C_{1} X-C_{2} X^{2}-C_{3} X^{3} .
\end{gathered}
$$




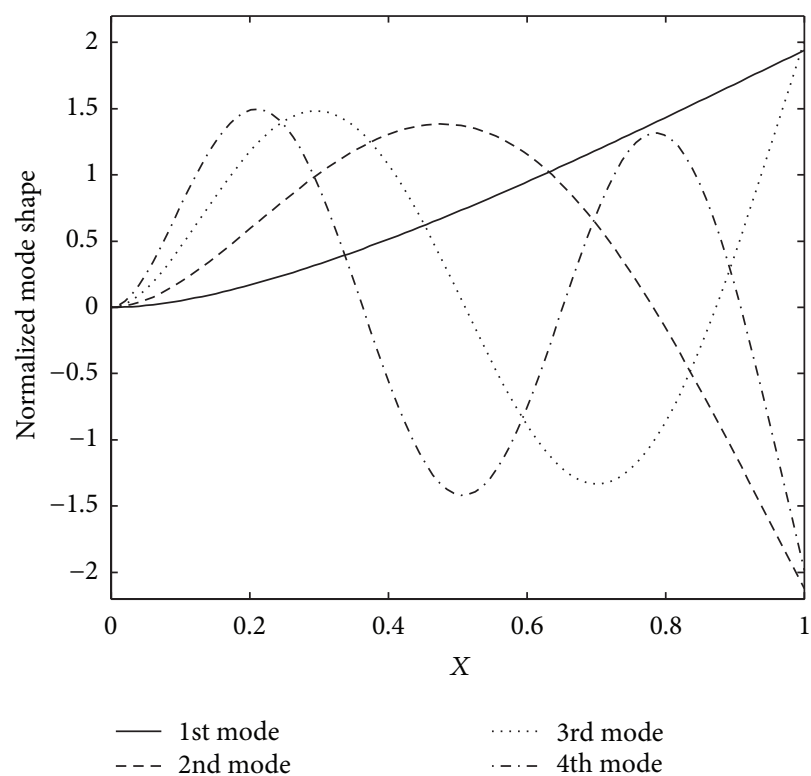

(a)

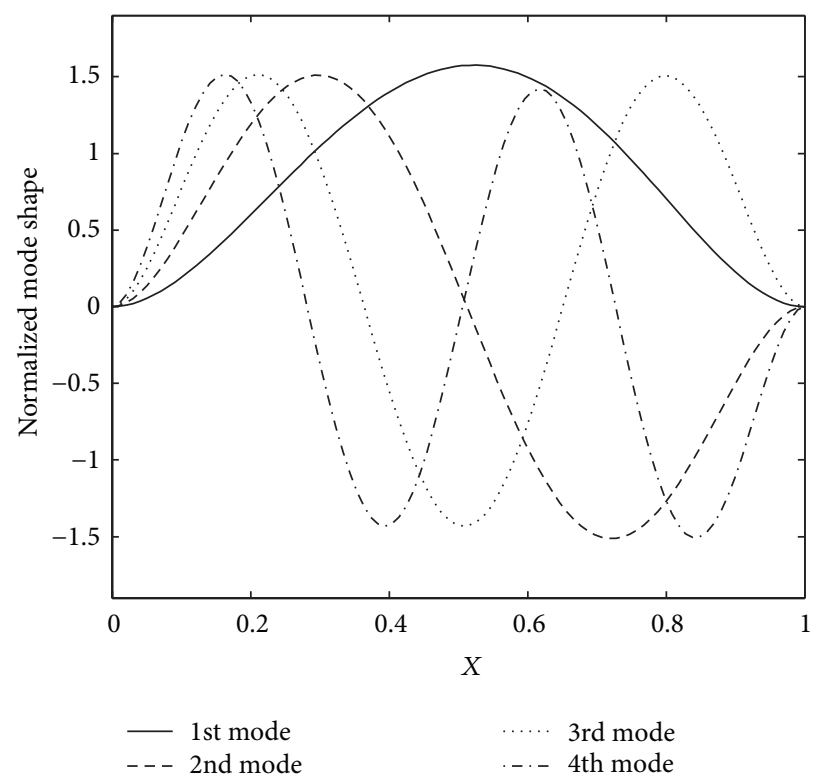

(b)

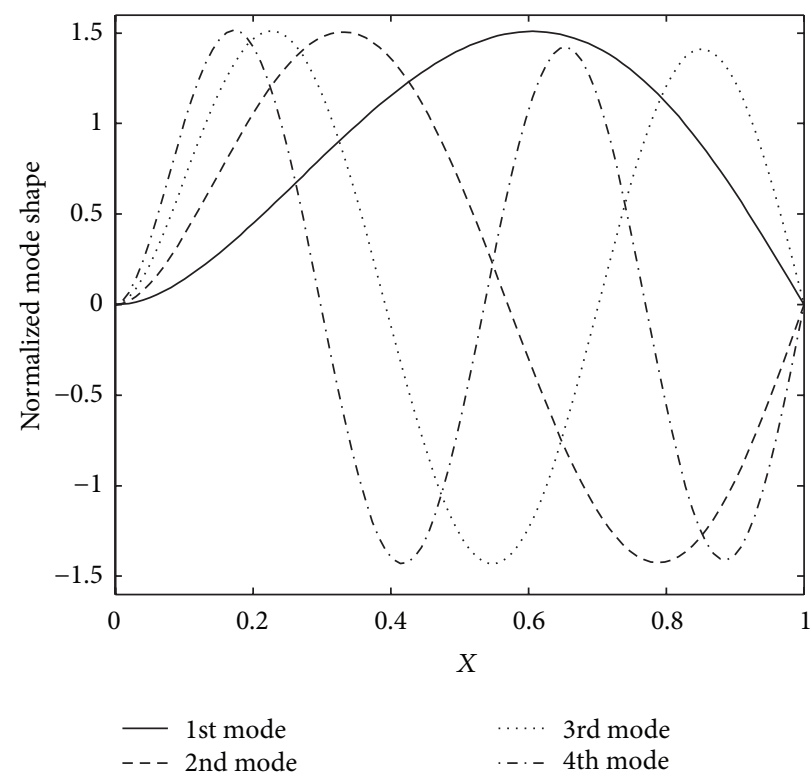

(c)

FIGURE 3: The first four normalized mode shapes for the (a) clamped-free beam, (b) clamped-clamped beam, and (c) clamped-simply supported beam when dimensionless rotating speed $U=4$ and offset length $R=3$.

Applying both sides of (5) with $G^{-1}$, we get the following:

$$
\begin{aligned}
G^{-1} G[\Phi(X)]=-G^{-1}\{ & -0.5 U^{2}(1+2 R) \frac{d^{2} \Phi(X)}{d X^{2}} \\
& +U^{2} R \frac{d}{d X}\left[X \frac{d \Phi(X)}{d X}\right]+0.5 U^{2} \\
& \left.\times \frac{d}{d X}\left[X^{2} \frac{d \Phi(X)}{d X}\right]-\lambda^{2} \Phi(X)\right\} .
\end{aligned}
$$

Substituting (6) and (8) into (9), we get the following:

$$
\begin{aligned}
\Phi(X)= & \sum_{m=0}^{3} C_{m} X^{m} \\
& +\sum_{m=0}^{\infty} \frac{0.5 U^{2}(1+2 R)(m+1)(m+2) C_{m+2}}{(m+1)(m+2)(m+3)(m+4)} X^{m+4} \\
& -\sum_{m=0}^{\infty} \frac{(m+1)^{2} U^{2} R C_{m+1}}{(m+1)(m+2)(m+3)(m+4)} X^{m+4}
\end{aligned}
$$




$$
\begin{aligned}
& -\sum_{m=0}^{\infty} \frac{0.5 U^{2} m(m+1) C_{m}}{(m+1)(m+2)(m+3)(m+4)} X^{m+4} \\
& +\frac{\lambda^{2} C_{m}}{(m+1)(m+2)(m+3)(m+4)} X^{m+4} .
\end{aligned}
$$

Finally, the coefficients $C_{m}$ in (10) can be determined by using the following recurrence relations:

$$
\begin{gathered}
C_{0}=\Phi(0), \quad C_{1}=\frac{d \Phi(0)}{d X} \\
C_{2}=\frac{1}{2} \frac{d^{2} \Phi(0)}{d X^{2}}, \quad C_{3}=\frac{1}{6} \frac{d^{3} \Phi(0)}{d X^{3}} \\
C_{m+4}=\frac{0.5 U^{2}(1+2 R) C_{m+2}}{(m+3)(m+4)}-\frac{(m+1) U^{2} R C_{m+1}}{(m+2)(m+3)(m+4)} \\
-\frac{0.5 U^{2} m C_{m}}{(m+2)(m+3)(m+4)} \\
+\frac{\lambda^{2} C_{m}}{(m+1)(m+2)(m+3)(m+4)}, \quad m \geq 0 .
\end{gathered}
$$

We may approximate the above solution by the $M$-term truncated series, and (6) can be rewritten as follows:

$$
\Phi(X)=\sum_{m=0}^{M} C_{m} X^{m} .
$$

Equation (13) implies that $\sum_{m=M+1}^{\infty} C_{m} X^{m}$ is negligibly small. The number of the series summation limit $M$ is determined by convergence requirement in practice.

From the above analysis, it can be found that there are five unknown parameters $\left(C_{0}, C_{1}, C_{2}, C_{3}\right.$, and $\left.\lambda\right)$ for the free vibration analysis of the rotating beam. These unknown parameters can be determined by using the boundary condition equations of the beam, and then the natural frequencies and corresponding mode shapes for the rotating beams can be obtained.

\section{Natural Frequencies and Mode Shapes}

The boundary conditions of the rotating beam shown in Figure 1 can be expressed into dimensionless form [1-4], and we get the following:

$$
\begin{array}{ll}
\frac{d^{2} \Phi(0)}{d X^{2}}-K_{L 1} \frac{d \Phi(0)}{d X}=0, & \frac{d^{3} \Phi(0)}{d X^{3}}+K_{L 0} \Phi(0)=0 \\
\frac{d^{2} \Phi(1)}{d X^{2}}+K_{R 1} \frac{d \Phi(1)}{d X}=0, & \frac{d^{3} \Phi(1)}{d X^{3}}-K_{R 0} \Phi(1)=0
\end{array}
$$

where $K_{L 1}=k_{L 1} L / E I, K_{L 0}=k_{L 0} L^{3} / E I, K_{R 1}=k_{R 1} L / E I$, $K_{R 0}=k_{R 0} L^{3} / E I, k_{L 0}$ and $k_{R 0}$ are the stiffness of the

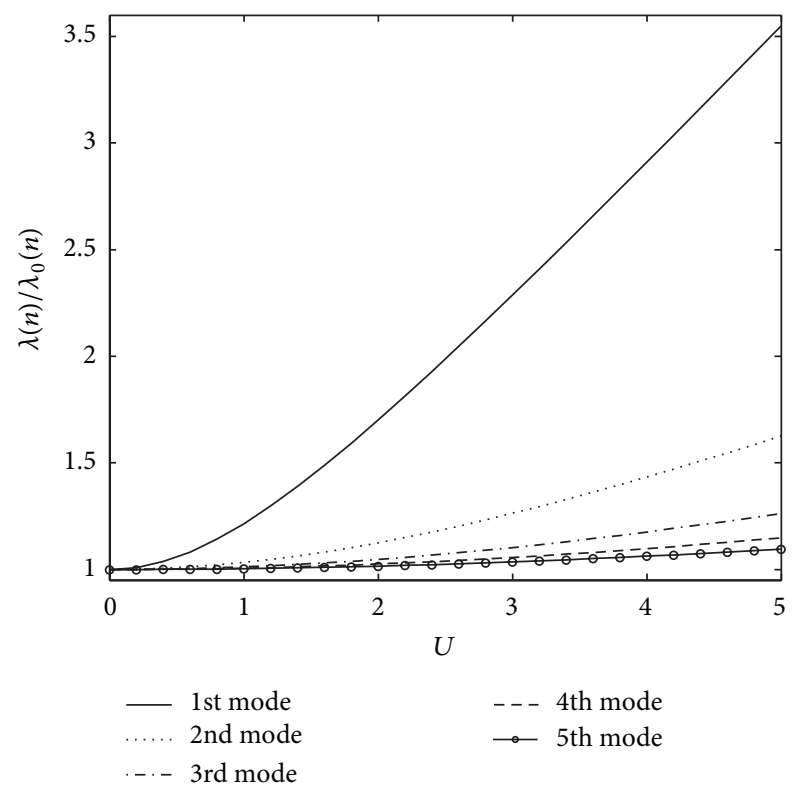

FIgURE 4: The first five dimensionless natural frequency ratios $\lambda(n) / \lambda_{0}(n)$ for (a) the clamped-free beam with various dimensionless rotating speeds (offset length $R=3$ ).

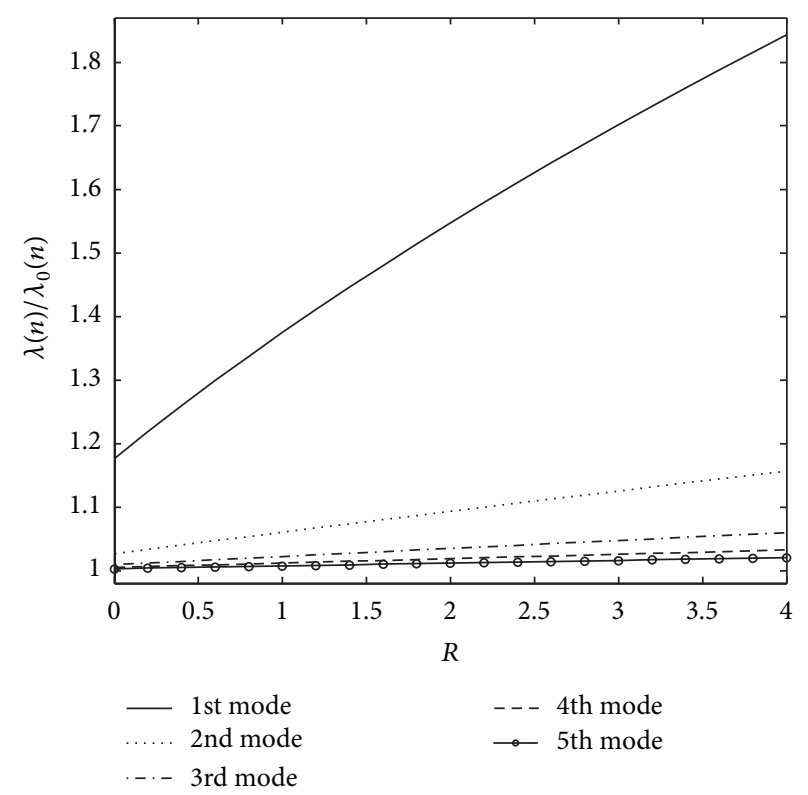

FIgURE 5: The first five dimensionless natural frequency ratios $\lambda(n) / \lambda_{0}(n)$ for the clamped-free beam with various dimensionless offset lengths (rotating speed $U=2$ ).

translational springs, and $k_{L 1}$ and $k_{R 1}$ are the stiffness of the rotational springs at $x=0$ and $L$, respectively.

Substituting (11) into (14), we get the following:

$$
C_{2}=K_{L 1} C_{1}, \quad C_{3}=-K_{L 0} C_{0} .
$$


TABLE 1: The first five dimensionless natural frequencies $\lambda(n)$ for a clamped-free beam with different dimensionless rotating speeds $U$ and offset lengths $R$.

\begin{tabular}{|c|c|c|c|c|c|c|c|}
\hline \multirow{2}{*}{$R$} & \multirow{2}{*}{$U$} & \multirow{2}{*}{ Methods } & \multicolumn{5}{|c|}{ Mode index $n$} \\
\hline & & & 1 & 2 & 3 & 4 & 5 \\
\hline \multirow{10}{*}{0} & \multirow{2}{*}{0} & Present & 3.516015 & 22.034492 & 61.697214 & 120.901916 & 199.859530 \\
\hline & & {$[4]$} & 3.5160 & 22.0345 & 61.6972 & 120.902 & 199.860 \\
\hline & \multirow{2}{*}{1} & Present & 3.681647 & 22.181011 & 61.841763 & 121.050922 & 200.011574 \\
\hline & & {$[3]$} & 3.6816 & 22.1810 & 61.8418 & - & - \\
\hline & \multirow{2}{*}{2} & Present & 4.137320 & 22.614922 & 62.273184 & 121.496695 & 200.466923 \\
\hline & & {$[3]$} & 4.1373 & 22.6149 & 62.2732 & - & - \\
\hline & \multirow{2}{*}{3} & Present & 4.797279 & 23.320264 & 62.984967 & 122.235547 & 201.223245 \\
\hline & & {$[3]$} & 4.7973 & 23.3203 & 62.9850 & - & - \\
\hline & \multirow{2}{*}{12} & Present & 13.170150 & 37.603112 & 79.614478 & 140.534354 & 220.536322 \\
\hline & & [4] & 13.1702 & 37.6031 & 79.6145 & 140.534 & 220.536 \\
\hline \multirow{10}{*}{1} & \multirow{2}{*}{1} & Present & 3.888824 & 22.375014 & 62.043053 & 121.263205 & 200.230870 \\
\hline & & {$[3]$} & 3.8888 & 22.3750 & 62.0431 & - & - \\
\hline & \multirow{2}{*}{2} & Present & 4.833689 & 23.366042 & 63.067548 & 122.339546 & 201.340072 \\
\hline & & {$[3]$} & 4.8337 & 23.3660 & 63.0675 & - & - \\
\hline & \multirow{2}{*}{4} & Present & 7.475048 & 26.957262 & 66.986772 & 126.537325 & 205.706683 \\
\hline & & {$[3]$} & 7.475 & 26.9573 & 66.9868 & - & - \\
\hline & \multirow{2}{*}{8} & Present & 13.507389 & 37.953793 & 80.529532 & 141.877971 & 222.165291 \\
\hline & & {$[3]$} & 13.5074 & 37.9538 & 80.5295 & - & - \\
\hline & \multirow{2}{*}{15} & Present & 24.409202 & 61.437052 & 113.488866 & 182.695785 & 268.831067 \\
\hline & & {$[1]$} & 24.4092 & 61.4371 & 113.4889 & - & - \\
\hline \multirow{6}{*}{2} & \multirow{2}{*}{1} & Present & 4.085335 & 22.567257 & 62.243575 & 121.475038 & 200.449876 \\
\hline & & {$[3]$} & 4.0853 & 22.5673 & 62.2436 & - & - \\
\hline & \multirow{2}{*}{2} & Present & 5.439949 & 24.092373 & 63.850233 & 123.175402 & 202.208659 \\
\hline & & {$[3]$} & 5.4399 & 24.0924 & 63.8502 & - & - \\
\hline & \multirow{2}{*}{4} & Present & 8.966379 & 29.380507 & 69.852259 & 129.712377 & 209.068207 \\
\hline & & {$[3]$} & 8.9664 & 29.3805 & 69.8523 & - & - \\
\hline \multirow{6}{*}{3} & \multirow{2}{*}{1} & Present & 4.272659 & 22.757784 & 62.443336 & 121.686425 & 200.668594 \\
\hline & & {$[3]$} & 4.2727 & 22.7578 & 62.4433 & - & - \\
\hline & \multirow{2}{*}{2} & Present & 5.983713 & 24.796124 & 64.621696 & 124.004411 & 203.072744 \\
\hline & & {$[3]$} & 5.9837 & 24.7961 & 64.6217 & - & - \\
\hline & \multirow{2}{*}{4} & Present & 10.236798 & 31.604901 & 72.583135 & 132.794372 & 212.364626 \\
\hline & & [1] & 10.2368 & 31.6049 & 72.5831 & - & - \\
\hline
\end{tabular}

Substituting (12) and (16) into (15), then (15) can be expressed as a linear function of $C_{0}$ and $C_{1}$ :

$$
\begin{aligned}
& \sum_{m=0}^{M}(m+1)(m+2) C_{m+2}+K_{R 1} \sum_{m=0}^{M}(m+1) C_{m+1} \\
& \quad=f_{11}(\lambda) C_{0}+f_{12}(\lambda) C_{1}=0 \\
& \sum_{m=0}^{M}(m+1)(m+2)(m+3) C_{m+3}-K_{R 0} \sum_{m=0}^{M} C_{m} \\
& \quad=f_{21}(\lambda) C_{0}+f_{22}(\lambda) C_{1}=0 .
\end{aligned}
$$

From (17) and (18), the $n$th dimensionless frequency parameter $\lambda(n)$ can be solved by the following:

$$
f_{11}(\lambda) f_{22}(\lambda)-f_{12}(\lambda) f_{21}(\lambda)=\sum_{n=0}^{N} S_{n} \lambda^{n}=0
$$

where $N$ is the greatest power of $\lambda$.

Notice that (19) is a polynomial of degree $N$ evaluated at $\lambda$. By using the functions sym2poly and roots in MATLAB Symbolic Math Toolbox, (19) can be directly solved. The next step is to determine the $n$th mode shape function corresponding to $n$th dimensionless frequency $\lambda(n)$. Substituting the solved 

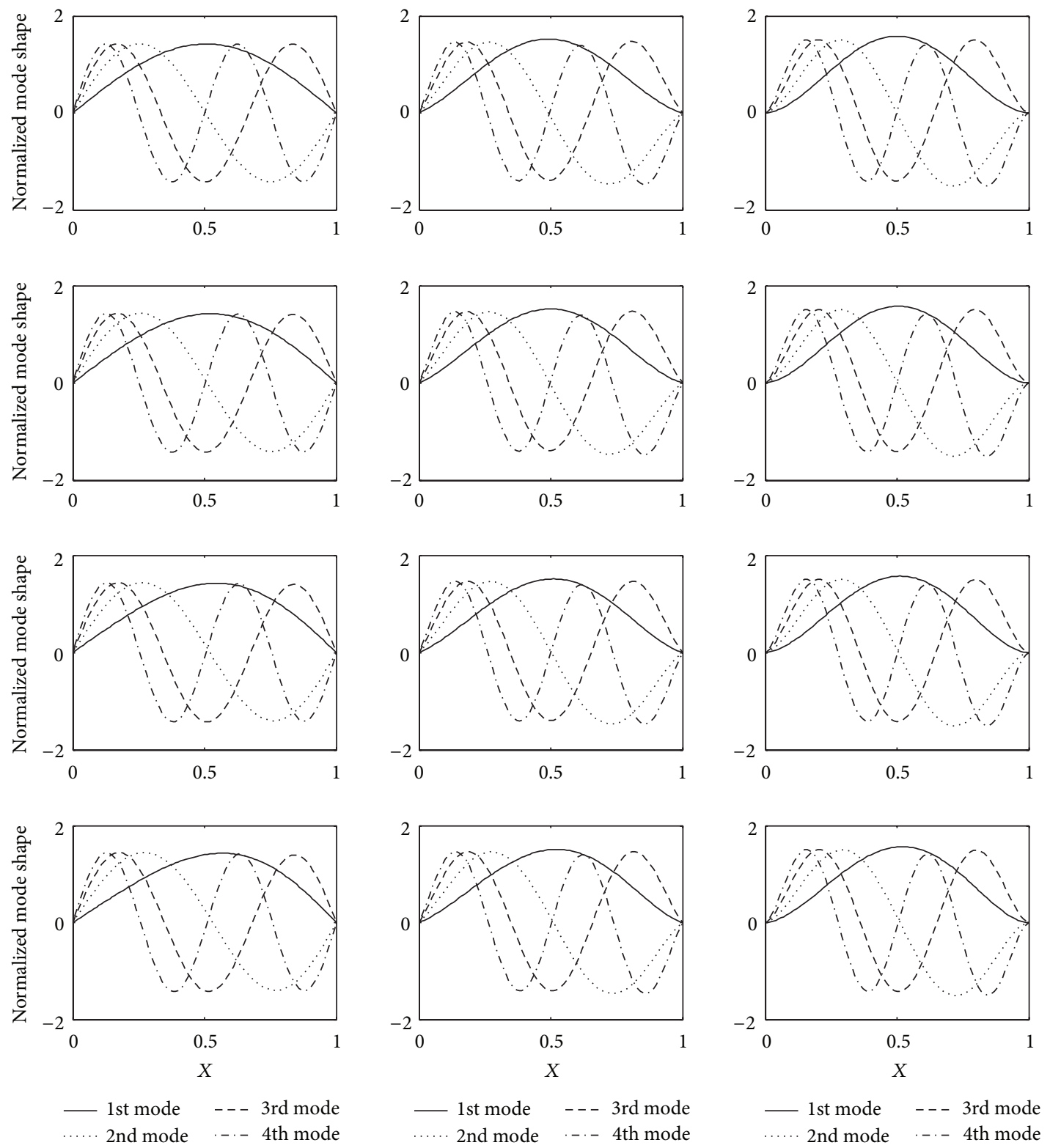

FIGURE 6: The first four normalized mode shapes for the rotating beams listed in Table 4. Columns 1, 2, and 3 are $\left(K_{L 1}=K_{R 1}=0\right),\left(K_{L 1}=10\right.$; $\left.K_{R 1}=20\right)$, and $\left(K_{L 1}=100 ; K_{R 1}=200\right)$, respectively. Rows $1,2,3$, and 4 are $U=1,2,3$, and 4, respectively.

$\lambda(n)$ into (17) or (18), $C_{1}$ can be expressed as the function of $C_{0}$ :

$$
C_{1}=-\frac{f_{11}(\lambda)}{f_{12}(\lambda)} C_{0}=-\frac{f_{21}(\lambda)}{f_{22}(\lambda)} C_{0}
$$

Substituting (11), (12), and (20) into (13), then the mode shape function can be obtained. By normalizing (13), the normalized mode shape is defined as follows:

$$
\bar{\Phi}(X)=\frac{\Phi(X)}{\sqrt{\int_{0}^{1}[\Phi(X)]^{2} d X}} .
$$

It can be found that the mode shape function by using AMDM is a continuous function (closed-form series solution) and not discrete numerical values at knot point by finite element or finite difference methods.

\section{Results and Discussion}

In order to verify the proposed method to analyze the free vibration of the rotating beam shown in Figure 1, several numerical examples will be discussed in this section.

As mentioned earlier, the closed-form series solutions of mode shape functions in (13) will have to be truncated in numerical calculations. It is important to check how rapidly the dimensionless natural frequencies $\lambda(n)$ computed 
TABLE 2: The first five dimensionless natural frequencies $\lambda(n)$ for a clamped-clamped beam with different dimensionless rotating speeds $U$ and offset lengths $R$.

\begin{tabular}{|c|c|c|c|c|c|c|c|}
\hline \multirow{2}{*}{$R$} & \multirow{2}{*}{$U$} & \multirow{2}{*}{ Methods } & \multicolumn{5}{|c|}{ Mode index $n$} \\
\hline & & & 1 & 2 & 3 & 4 & 5 \\
\hline \multirow{8}{*}{0} & \multirow{2}{*}{0} & Present & 22.373285 & 61.672823 & 120.903392 & 1.998594481274627 & 2.985555379664196 \\
\hline & & [3] & 22.3733 & 61.6728 & 120.9034 & - & - \\
\hline & \multirow{2}{*}{1} & Present & 22.465244 & 61.801647 & 121.044116 & 200.006494 & 298.706437 \\
\hline & & {$[3]$} & 22.4652 & 61.8016 & 121.0441 & - & - \\
\hline & \multirow{2}{*}{2} & Present & 22.738323 & 62.186191 & 121.465166 & 200.446898 & 299.158620 \\
\hline & & {$[3]$} & 22.7383 & 62.1862 & 121.4652 & - & - \\
\hline & \multirow{2}{*}{4} & Present & 23.791502 & 63.696418 & 123.132818 & 202.197641 & 300.959712 \\
\hline & & {$[3]$} & 23.7915 & 63.6964 & 123.1328 & - & - \\
\hline \multirow{6}{*}{1} & \multirow{2}{*}{1} & Present & 22.601469 & 61.987491 & 121.248139 & 200.220805 & 298.927273 \\
\hline & & {$[3]$} & 22.6015 & 61.9875 & 121.2481 & - & - \\
\hline & \multirow{2}{*}{2} & Present & 23.269013 & 62.919871 & 122.275462 & 201.300307 & 300.039256 \\
\hline & & {$[3]$} & 23.2690 & 62.9199 & 122.2755 & - & - \\
\hline & \multirow{2}{*}{4} & Present & 25.721997 & 66.488904 & 126.285440 & 205.551660 & 304.439765 \\
\hline & & {$[3]$} & 25.7220 & 66.4889 & 126.2854 & - & - \\
\hline \multirow{6}{*}{2} & \multirow{2}{*}{1} & Present & 22.736642 & 62.172635 & 121.451744 & 200.434838 & 299.147913 \\
\hline & & {$[3]$} & 22.7366 & 62.1726 & 121.4517 & - & - \\
\hline & \multirow{2}{*}{2} & Present & 23.784414 & 63.642885 & 123.079231 & 202.149350 & 300.916791 \\
\hline & & {$[3]$} & 23.7844 & 63.6429 & 123.0792 & - & - \\
\hline & \multirow{2}{*}{4} & Present & 27.477304 & 69.138595 & 129.343258 & 208.839952 & 307.872284 \\
\hline & & {$[3]$} & 27.4773 & 69.1386 & 129.3433 & - & - \\
\hline \multirow{6}{*}{3} & \multirow{2}{*}{1} & Present & 22.870783 & 62.3570858594266 & 121.6549319912815 & 200.6485958268648 & 299.3683574772145 \\
\hline & & {$[3]$} & 22.8708 & 62.3571 & 121.6549 & - & - \\
\hline & \multirow{2}{*}{2} & Present & 24.285626 & 64.355627 & 123.876600 & 202.994081 & 301.791251 \\
\hline & & {$[3]$} & 24.2856 & 64.3556 & 123.8766 & - & - \\
\hline & \multirow{2}{*}{4} & Present & 29.093946 & 71.663126 & 132.313123 & 212.065591 & 311.259430 \\
\hline & & {$[3]$} & 29.0939 & 71.6631 & 132.3131 & - & - \\
\hline
\end{tabular}

through AMDM converge toward the exact value as the series summation limit $M$ is increased. To examine the convergence of the solution, a clamped-free beam with dimensionless rotating speed $U=4$ and dimensionless offset length $R=3$ is considered. In this study, the classical boundary conditions (such as clamped, simply supported, and free) can be considered as the special cases of (14) and (15). For example, the clamped boundary condition is obtained by setting the stiffness of the translational and rotational springs to be extremely large (which is represented by a very large number, $1 \times 10^{9}$, in this paper). Similarly, for simply supported boundary condition, the stiffness of the translational and rotational springs is set to $1 \times 10^{9}$ and 0 , respectively. For free boundary condition, the stiffness of the translational and rotational springs is set to 0 . Figure 2 shows the first five dimensionless natural frequencies $\lambda(n)$ as the function of the series summation limit $M$. Clearly, the $\lambda(n)$ converges very quickly as the series summation limit $M$ is increased. The excellent numerical stability of the solution can also be found in Figure 2.

For brief, the series summation limit $M$ in (13) will be simply truncated to $M=60$ in all the subsequent calculations. The dimensionless natural frequencies $\lambda(n)$ are kept accurate to the sixth decimal place for comparison purpose. Tables 1, 2, and 3 list the first five dimensionless natural frequencies $\lambda(n)$ of the beam under various dimensionless rotating speeds $U$ and offset lengths $R$ for clamped-free, clamped-clamped, and clamped-simply supported boundary conditions, respectively. Those calculated results are compared with those listed in $[1,3,4]$, and excellent agreement is found. Figure 3 shows the first four normalized mode shapes for different boundary conditions when dimensionless rotating speed $U=4$ and offset length $R=3$.

Figures 4 and 5 show the first five dimensionless natural frequency ratios $\lambda(n) / \lambda_{0}(n)$ for the clamped-free beam as the functions of the dimensionless rotating speed $U$ and offset length $R$, where $\lambda_{0}(n)$ is the corresponding dimensionless natural frequencies when $U=0$ (nonrotating beam). From Figures 4 and 5, it can be found that the natural frequencies' ratios increase when the rotating speed or offset length increases for both beams. However, the variations on the natural frequency ratios of the low order modes are more sensitive to the rotating speed or offset length.

Next, the beams with general boundary conditions are discussed. Because the proposed method based on AMDM technique offers a unified and systematic procedure for 
TABLE 3: The first five dimensionless natural frequencies $\lambda(n)$ for a clamped-simply supported beam with different dimensionless rotating speeds $U$ and offset lengths $R$.

\begin{tabular}{|c|c|c|c|c|c|c|c|}
\hline \multirow{2}{*}{$R$} & \multirow{2}{*}{$U$} & \multirow{2}{*}{ Methods } & \multicolumn{5}{|c|}{ Mode index $n$} \\
\hline & & & 1 & 2 & 3 & 4 & 5 \\
\hline \multirow{8}{*}{0} & \multirow{2}{*}{0} & Present & 15.418206 & 49.964862 & 104.247696 & 178.269729 & 272.030971 \\
\hline & & {$[3]$} & 15.4182 & 49.9649 & 104.2477 & - & - \\
\hline & \multirow{2}{*}{1} & Present & 15.512970 & 50.093465 & 104.388569 & 178.416902 & 272.181974 \\
\hline & & {$[3]$} & 15.5130 & 50.0935 & 104.3886 & - & - \\
\hline & \multirow{2}{*}{2} & Present & 15.793333 & 50.476967 & 104.809884 & 178.857595 & 272.634416 \\
\hline & & {$[3]$} & 15.7933 & 50.4770 & 104.8099 & - & - \\
\hline & \multirow{2}{*}{4} & Present & 16.861201 & 51.977798 & 106.475988 & 180.608162 & 274.435777 \\
\hline & & {$[3]$} & 16.8612 & 51.9778 & 106.4760 & - & - \\
\hline \multirow{6}{*}{1} & \multirow{2}{*}{1} & Present & 15.650431 & 50.276757 & 104.591434 & 178.630496 & 272.402329 \\
\hline & & {$[3]$} & 15.6504 & 50.2768 & 104.5914 & - & - \\
\hline & \multirow{2}{*}{2} & Present & 16.324050 & 51.198754 & 105.614686 & 179.707690 & 273.512869 \\
\hline & & {$[3]$} & 16.3240 & 51.1988 & 105.6147 & - & - \\
\hline & \multirow{2}{*}{4} & Present & 18.739775 & 54.700871 & 109.594297 & 183.942227 & 277.903130 \\
\hline & & {$[3]$} & 18.7398 & 54.7009 & 109.5943 & - & - \\
\hline \multirow{6}{*}{2} & \multirow{2}{*}{1} & Present & 15.786476 & 50.459230 & 104.793819 & 178.843781 & 272.622470 \\
\hline & & {$[3]$} & 15.7865 & 50.4592 & 104.7938 & - & - \\
\hline & \multirow{2}{*}{2} & Present & 16.834938 & 51.908185 & 106.412044 & 180.552930 & 274.387932 \\
\hline & & {$[3]$} & 16.8349 & 51.9082 & 106.4120 & - & - \\
\hline & \multirow{2}{*}{4} & Present & 20.412962 & 57.264050 & 112.606262 & 187.203776 & 281.318676 \\
\hline & & {$[3]$} & 20.4130 & 57.2641 & 112.6063 & - & - \\
\hline \multirow{6}{*}{3} & \multirow{2}{*}{1} & Present & 15.921144 & 50.640895 & 104.995728 & 179.056759 & 272.842396 \\
\hline & & {$[3]$} & 15.9211 & 50.6409 & 104.9957 & - & - \\
\hline & \multirow{2}{*}{2} & Present & 17.327885 & 52.605804 & 107.202130 & 181.393382 & 275.259636 \\
\hline & & {$[3]$} & 17.3279 & 52.6058 & 107.2021 & - & - \\
\hline & \multirow{2}{*}{4} & Present & 21.932288 & 59.690141 & 115.520706 & 190.396605 & 284.684295 \\
\hline & & {$[3]$} & 21.9323 & 59.6901 & 115.5207 & - & - \\
\hline
\end{tabular}

vibration analysis, the modification of boundary conditions from one case to another is as simple as changing the values of the stiffness of translational and rotational springs. And it does not involve any changes to the solution procedures or algorithms.

Table 4 lists the first five dimensionless natural frequency $\lambda(n)$ for the beam with different dimensionless rotating speeds $U$ and different rotational springs $K_{L 1}$ and $K_{R 1}$ when the translational springs $K_{L 0}=K_{R 0}=1 \times 10^{9}$ and the dimensionless offset length $R=3$. From Table 4 , it is found that the natural frequencies increase when the offset length or rotating speed increases, as expected. Figure 6 shows the first four normalized mode shapes of the rotating beam listed in Table 4. From Figure 6, it can be found that the discrepancies of the mode shapes under different rotating speeds are very small. However, the natural frequencies are quite different, as shown in Table 4.

Based on the developments achieved and results obtained in this paper, the following remarks can be made.

(1) The essential steps of the AMDM application includes transforming the governing differential equation for the rotating beam into algebraic equation; by using the boundary condition equations, any desired dimensionless natural frequencies and corresponding mode shapes can be easily obtained simultaneously.

(2) All the steps of the AMDM are very straightforward, and the application of the AMDM to both equations of motion and the boundary conditions seems to be very involved computationally. However, all the algebraic calculations are finished quickly using symbolic computational software (such as MATLAB). Besides all these, the analysis of the convergence of the results shows that AMDM solutions converge fast. The results of the AMDM are found in excellent agreement with available published results.

\section{Conclusions}

In this paper, free vibrations of the uniform rotating Euler-Bernoulli beams under different boundary conditions are analyzed using Adomian modified decomposition method (AMDM). The advantages of the AMDM are its fast convergence of the solution and its high degree of accuracy. Natural frequencies and corresponding mode shapes with various boundary conditions, dimensionless offset length, and dimensionless rotating speed are presented. 
TABLE 4: The first five dimensionless natural frequencies $\lambda(n)$ for the beam with different dimensionless offset lengths $R$ and rotational spring stiffness $K_{L 1}$ and $K_{R 1}$ when the translational spring stiffness $K_{L 0}=K_{R 0}=1 \times 10^{9}$ and the dimensionless offset length $R=3$.

\begin{tabular}{|c|c|c|c|c|c|c|c|}
\hline \multirow{2}{*}{$U$} & \multirow{2}{*}{$K_{L 1}$} & \multirow{2}{*}{$K_{R 1}$} & \multicolumn{5}{|c|}{ Mode index $n$} \\
\hline & & & 1 & 2 & 3 & 4 & 5 \\
\hline \multirow{3}{*}{1} & 0 & 0 & 10.728125 & 40.379864 & 89.736290 & 158.826493 & 247.654314 \\
\hline & 10 & 20 & 18.722928 & 52.557379 & 104.894264 & 176.148714 & 266.614189 \\
\hline & 100 & 200 & 22.240871 & 60.660903 & 118.406571 & 195.403654 & 291.714050 \\
\hline \multirow{3}{*}{2} & 0 & 0 & 12.901808 & 42.950832 & 92.403230 & 161.529064 & 250.373747 \\
\hline & 10 & 20 & 20.251238 & 54.708735 & 107.278904 & 178.653087 & 269.187647 \\
\hline & 100 & 200 & 23.660610 & 62.669418 & 120.641412 & 197.764434 & 294.153770 \\
\hline \multirow{3}{*}{3} & 0 & 0 & 15.741169 & 46.861509 & 96.655335 & 165.918965 & 254.831087 \\
\hline & 10 & 20 & 22.506559 & 58.074886 & 111.115686 & 182.735812 & 273.412896 \\
\hline & 100 & 200 & 25.806448 & 65.847359 & 124.257999 & 201.625455 & 298.167186 \\
\hline \multirow{3}{*}{4} & 0 & 0 & 18.856444 & 51.730723 & 102.251676 & 171.841633 & 260.921080 \\
\hline & 10 & 20 & 25.233188 & 62.406770 & 116.227213 & 188.272249 & 279.199963 \\
\hline & 100 & 200 & 28.463584 & 69.993005 & 129.113530 & 206.884873 & 303.679326 \\
\hline
\end{tabular}

Furthermore, the natural frequencies obtained by using AMDM are in excellent agreement with published results.

It should be noted that the proposed method can be used to analyze the vibration of the rotating beams under arbitrary boundary conditions. The vibration analysis for different boundary conditions and/or rotating speed is as simple as changing the value of corresponding parameters and does not involve any changes to the solution procedures or algorithms.

The results in this paper show that the AMDM technique is reliable, powerful, and promising for solving free vibration problems for rotating beams. The author believes that the AMDM can further be applied to the Timoshenko rotating beam problems and also it can be used as an alternative to other solution techniques such as finite element method, differential quadrature method, and Frobenius method.

\section{Acknowledgments}

This work was partly sponsored by the National Natural Science Foundation of China (no. 51265037), Scientific Research Foundation for the Returned Overseas Chinese Scholars, State Education Ministry (no. 2012-44), and Technology Foundation of Jiangxi Province, China (no. KJLD12075).

\section{References}

[1] C. Mei, "Application of differential transformation technique to free vibration analysis of a centrifugally stiffened beam," Computers and Structures, vol. 86, no. 11-12, pp. 1280-1284, 2008.

[2] O. Özdemir and M. O. Kaya, "Flapwise bending vibration analysis of a rotating tapered cantilever Bernoulli-Euler beam by differential transform method," Journal of Sound and Vibration, vol. 289, no. 1-2, pp. 413-420, 2006.

[3] S. Naguleswaran, "Lateral vibration of a centrifugally tensioned uniform Euler-Bernoulli beam," Journal of Sound and Vibration, vol. 176, no. 5, pp. 613-624, 1994.

[4] G. Wang and N. M. Wereley, "Free vibration analysis of rotating blades with uniform tapers," AIAA Journal, vol. 42, no. 12, pp. 2429-2437, 2004.
[5] M. H. Tsai, W. Y. Lin, Y. C. Zhou, and K. M. Hsiao, "A corotational finite element method combined with floating frame method for large steady-state deformation and free vibration analysis of a rotating-inclined beam," Mathematical Problems in Engineering, vol. 2011, Article ID 146505, 29 pages, 2011.

[6] J. R. Banerjee, "Free vibration of centrifugally stiffened uniform and tapered beams using the dynamic stiffness method," Journal of Sound and Vibration, vol. 233, no. 5, pp. 857-875, 2000.

[7] K. G. Vinod, S. Gopalakrishnan, and R. Ganguli, "Free vibration and wave propagation analysis of uniform and tapered rotating beams using spectrally formulated finite elements," International Journal of Solids and Structures, vol. 44, no. 18-19, pp. 5875-5893, 2007.

[8] H. H. Yoo and S. H. Shin, "Vibration analysis of rotating cantilever beams," Journal of Sound and Vibration, vol. 212, no. 5, pp. 807-808, 1998.

[9] S. K. Das, P. C. Ray, and G. Pohit, "Free vibration analysis of a rotating beam with nonlinear spring and mass system," Journal of Sound and Vibration, vol. 301, no. 1-2, pp. 165-188, 2007.

[10] C. L. Huang, W. Y. Lin, and K. M. Hsiao, "Free vibration analysis of rotating Euler beams at high angular velocity," Computers and Structures, vol. 88, no. 17-18, pp. 991-1001, 2010.

[11] P. P. S. Chhabra and R. Ganguli, "Superconvergent finite element for coupled torsional-flexural-axial vibration analysis of rotating blades," International Journal for Computational Methods in Engineering Science and Mechanics, vol. 11, no. 1, pp. 48-69, 2010.

[12] D. Thakkar and R. Ganguli, "Dynamic response of rotating beams with piezoceramic actuation," Journal of Sound and Vibration, vol. 270, no. 4-5, pp. 729-753, 2004.

[13] P. K. Datta and R. Ganguli, "Vibration characteristics of a rotating blade with localized damage including the effects of shear deformation and rotary inertia," Computers and Structures, vol. 36, no. 6, pp. 1129-1133, 1990.

[14] G. Adomian, Solving Frontier Problems of Physics: The Decomposition Method, Kluwer Academic Publishers, Boston, Mass, USA, 1994.

[15] A.-M. Wazwaz, "Analytic treatment for variable coefficient fourth-order parabolic partial differential equations," Applied Mathematics and Computation, vol. 123, no. 2, pp. 219-227, 2001.

[16] T. Öziş and A. Yıldırım, "Comparison between Adomian's method and He's homotopy perturbation method," Computers 
\& Mathematics with Applications, vol. 56, no. 5, pp. 1216-1224, 2008.

[17] A.-M. Wazwaz and S. M. El-Sayed, "A new modification of the Adomian decomposition method for linear and nonlinear operators," Applied Mathematics and Computation, vol. 122, no. 3, pp. 393-405, 2001.

[18] J. C. Hsu, H. Y. Lai, and C. K. Chen, "Free vibration of nonuniform Euler-Bernoulli beams with general elastically end constraints using Adomian modified decomposition method," Journal of Sound and Vibration, vol. 318, no. 4-5, pp. 965-981, 2008.

[19] Q. Mao and S. Pietrzko, "Design of shaped piezoelectric modal sensor for beam with arbitrary boundary conditions by using Adomian decomposition method," Journal of Sound and Vibration, vol. 329, no. 11, pp. 2068-2082, 2010.

[20] Q. Mao, "Free vibration analysis of elastically connected multiple-beams by using the Adomian modified decomposition method," Journal of Sound and Vibration, vol. 331, no. 11, pp. 2532-2542, 2012.

[21] S. Das, "A numerical solution of the vibration equation using modified decomposition method," Journal of Sound and Vibration, vol. 320, no. 3, pp. 576-583, 2009. 


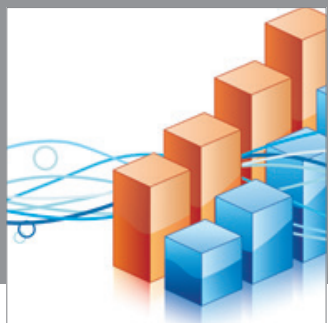

Advances in

Operations Research

mansans

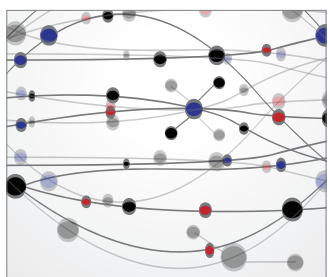

The Scientific World Journal
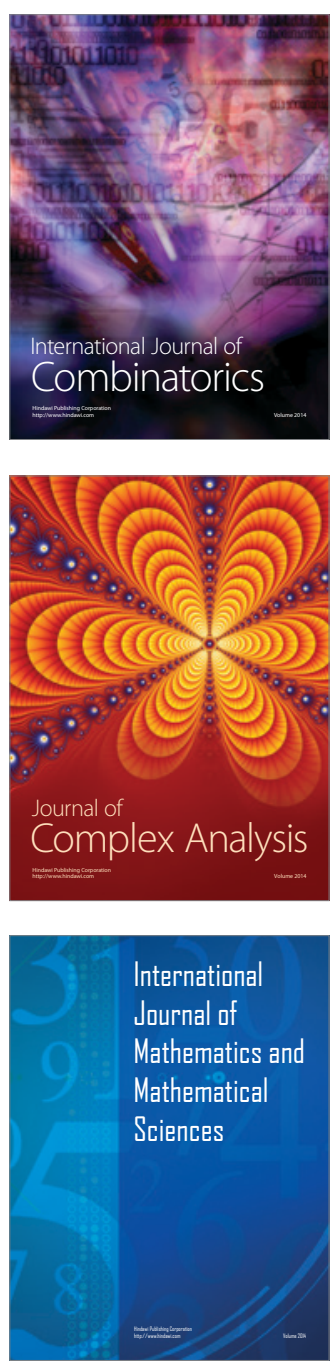
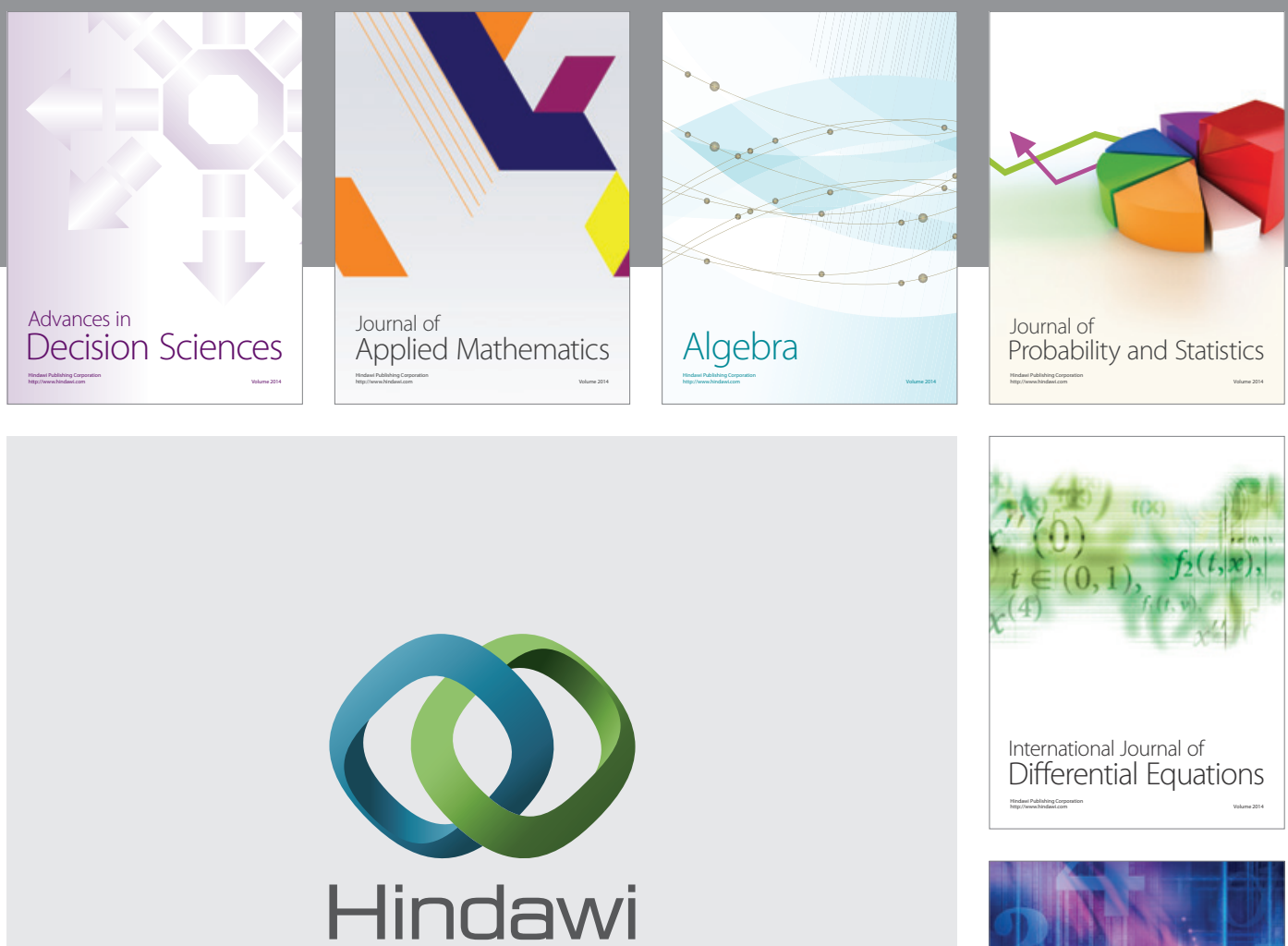

Submit your manuscripts at http://www.hindawi.com
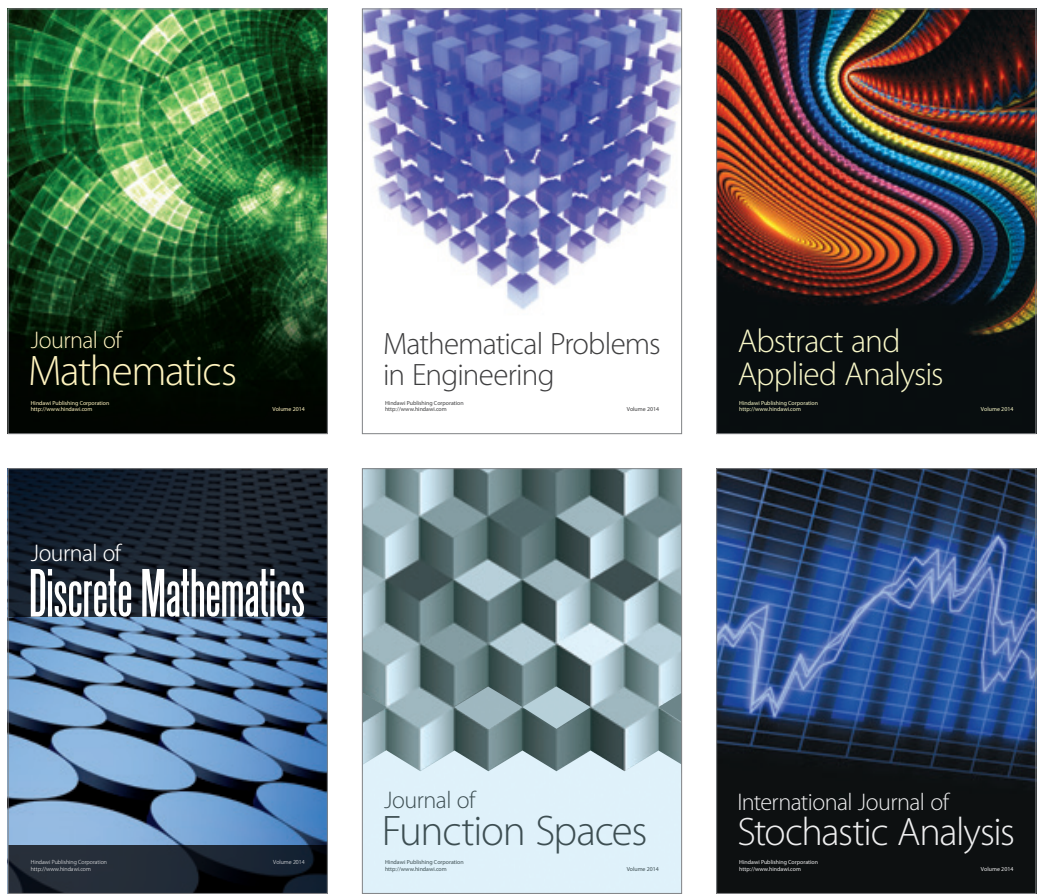

Journal of

Function Spaces

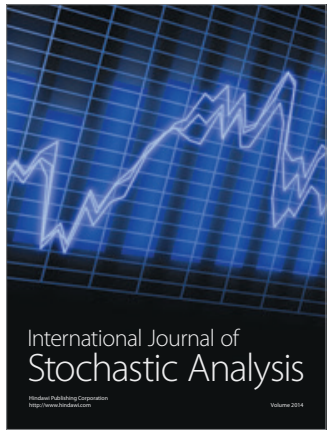

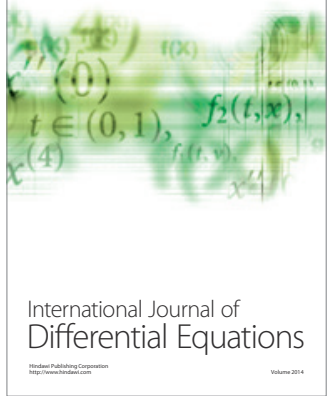
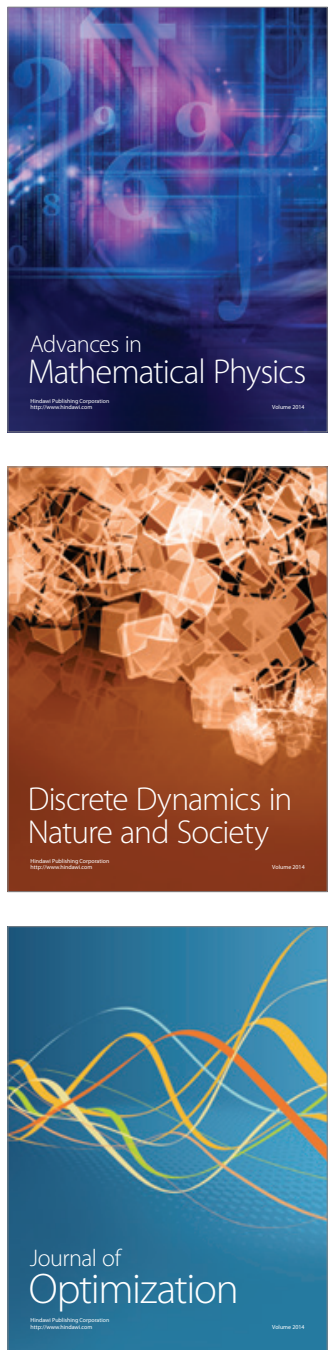